\title{
DIVERSIDAD CULTURAL EN LOS CURRÍCULOS DE PSICOLOGÍA
}

\section{Cultural diversity on program of Physichology}

\author{
Norma Maldonado Santiago, Ph.D.*, Laura M. Pietri, Ph.D. ** y \\ Nancy V. Lemos Ramírez, M.H.S***
}

\begin{abstract}
Resumen
Los cursos de diversidad cultural en los currículos de psicología deben lograr sensibilizaciones hacia esos Otros que pudieran provocar emociones negativas por ser diferentes. Los estudios universitarios y las prácticas correspondientes como académicos y profesionales se sostienen en un cuerpo hegemónico que responde a una cultura en particular con pretensión universalista. Se realizó un estudio cualitativo y se examinaron los sílabos de cursos que atienden el tema de diversidad en programas de psicología de universidades públicas y privadas en Puerto Rico, Europa y Latinoamérica. El análisis de contenido reveló que el acercamiento al tema responde típicamente a la idiosincrasia del país, a las circunstancias psicosociales y políticas particulares y a la mediación de organismos y agencias acreditadoras. Se discute la diversidad inherente a la psicología, tanto en su enseñanza como en su aplicación.
\end{abstract}

Palabras claves: Diversidad cultural, formación académica, psicología.

\begin{abstract}
The Cultural Diversity courses on Programn of Psychology should raise students' awareness towards Others who tend to provoke negative emotions because of being different. The university courses and professional practices seem to respond to a particular culture while pretending to be universal in their postures. A qualitative study was conducted in which syllabuses of cultural diversity courses corresponding to different academic institutions from Puerto Rico, Europe and Latin America were examined. The content analysis revealed that the approach to the subject typically corresponds to the country's idiosyncrasy, psychosocial circumstances and particular regulations as well as the intervention of organizations and accrediting agencies. It is discussed the inherent diversity to the psychology, even in their teaching as in its application.
\end{abstract}

Key words: Cultural diversity, academic programs, psychology.

* Psicóloga Clínica y Catedrática Asociada Pontificia Universidad Católica de Puerto Rico nmaldonado@email.pucpr.edu; norma514@prtc.net

** Psicóloga Clínica y Catedrática Asociada Pontificia Universidad Católica de Puerto Rico lauram4u@aol.com

*** Estudiante Doctoral Colegio de Estudios Graduados en Ciencias de la Conducta y Asuntos de la Comunidad. nancy.lemos@upb.edu.co; vivita99@hotmail.com 


\section{INTRODUCCIÓN}

En el continuo de vida que supone la historia, las personas atraviesan grandes transformaciones que apelan a la reflexión de sus actos. La Psicología, como disciplina de encuentro humano, acuña la acción de generar espacios que abonan a la construcción de saberes desde una postura metacognitiva. Si bien supone propiciarse en un ambiente de confianza, respeto y democracia para el enriquecimiento de las relaciones humanas, la disciplina no está ajena de sucumbir ante los diversos conflictos que se suscitan en todas las regiones del mundo. Muchas sociedades han multiplicado las tendencias hacia los caminos de la desigualdad, la fragmentación y las situaciones de exclusión y discriminación. Por lo que se hace pertinente dar espacio a la mirada pedagógica que aúne esfuerzos reflexivos en torno a las identidades y las diferencias. $\mathrm{Al}$ reconocer la manera en que las personas se perciben y se vinculan con los Otros, podremos abonar a la construcción de modos de relación que tengan como horizonte, la igualdad y la inclusión.

La diversidad es un hecho cotidiano, por lo que hoy día, su presencia es evidente, al insertarse en fenómenos sociales que antes eran realidades irrefutables y convenientemente uniformes. En la actualidad «existen alrededor de 6000 grupos étnicos con características espirituales, materiales y emocionales distintas»e insertados en estructuras sociales alrededor del mundo (Martínez, 2006) (p. 1). Lo anterior evidencia que tanto las condiciones de globalización, histórico-filosóficas, como socio-culturales, han propiciado corrientes que hacen imperativo, el llamado a ejercer una mirada crítica al complejo entramado social del que se es partícipe. Ciertamente, la diversidad cultural abre el crisol para considerar un amplio espectro de realidades que extienden sus implicaciones al quehacer psicológico. Realidades que deben asumirse, tomando en consideración el entendimiento de la óptica teórica y geográfica.

El propósito de este estudio se enmarcó en abordar el rol que asumen las universidades públicas y privadas, que ofrecen estudios de pre-grado en psicología, ante el tema de la diversidad cultural. Las propuestas curriculares recientes se fundamentan en el desarrollo de competencias profesionales, lo que propicia prestar atención de calidad a la diversidad, (Ortiz,
2007). La Psicología como disciplina orientada al estudio y entendimiento del quehacer humano, no puede apartarse de estas posturas conciliadoras e inclusivas. De modo que, la integración de cursos que contemplen la diversidad cultural dentro de los currículos de Psicología a nivel de pre-grado, debe ser concebida como una invitación a analizar críticamente los supuestos culturales hegemónicos de los que el ser humano es objeto, tanto de manera pasiva como activa. A su vez, posibilita la apertura a considerar el andamiaje de las diversas formas culturales con las que el individuo convive, tomando como punto de partida la referencia geográfica e histórica local al plano mundial y virtual (Miranda, 2006)

La educación superior formal debe lograr sensibilizaciones hacia esos Otros que nos provocan empatía, temor, sospecha, coraje, curiosidad. Los psicólogos y psicólogas apuntan a la construcción de visiones alternas para entender las realidades de vida, por lo que en el ejercicio de la formación académica, se exhorta al encuentro de espacios que posibiliten la reflexión, el cuestionamiento y la contemplación de nuevos saberes. Para esto, se hace necesario el apoderamiento de discursos que promuevan identidades sociales y prácticas políticas no discriminatorias. La Academia, en su rol de facilitar el andamiaje de distintas formas de saber y pensar, puede contribuir de manera significativa a este objetivo. Es un escenario idóneo para fortalecer las tendencias democráticas y pluralistas así como, para minar aquellas que se tornan excluyentes e intolerantes

Consistente con una postura amplia e inclusiva y considerando que el número de instituciones académicas que tienen dentro de su oferta curricular la educación superior en psicología en Latinoamérica y Europa es vasta, el estudio se realizó con universidades de Puerto Rico, América Latina y una de Europa. Se pretendió conocer hasta qué punto los estudios universitarios y las prácticas como académicos y profesionales se sostienen en un cuerpo hegemónico que responde a una cultura en particular con pretensión universalista. Asimismo, se exploró si estaban representadas en los cursos, múltiples explicaciones del quehacer humano o si por el contrario, quedaban excluidos algunos saberes y maneras de ver y vivir las realidades. 


\section{FUNDAMENTOS TEÓRICOS}

Desde un ejercicio responsable del quehacer psicológico, se aúnan esfuerzos hacia el apoyo y acompañamiento de los procesos propios de cada ser humano. Por lo mismo, el compromiso profesional debe dirigirse hacia la persona, en lugar de llevarlo al territorio y discurso del psicólogo o psicóloga. Es a partir de este encuentro, que se comienzan a generar espacios donde se construyen lazos caracterizados por la mutua escucha. Acción que se articula en la confluencia de pasajes fluidos hacia la concertación de entornos dignos que propicien la autonomía del individuo. La formación académica en psicología debe propiciar espacios para la reflexión en torno a las identidades y las diferencias, cómo nos percibimos a nosotros mismos, de qué manera nos vinculamos a los otros y cuáles son las vías para construir modos de relación que tengan como horizonte la igualdad y la inclusión.

Ciertamente, la psicología responde a las demandas y exigencias del colectivo, a la vez que subyace en la diversidad de posturas y actitudes. La academia puede contribuir de una manera significativa a fortalecer las tendencias democráticas, pluralistas y contrarrestar las excluyentes e intolerantes. Este objetivo debería atravesar los currículos académicos particularmente los de psicología.

La diversidad cultural no es solo un hecho natural, sugiere un amplio espectro de las realidades socioculturales con sus implicaciones psicológicas. Es una perspectiva integradora de entender lo psicológico como parte del tejido social que intenta entender las realidades sociales. Desde estas premisas se posibilita un quehacer psicológico que escudriñe actores pensantes, ávidos de encontrar algún sentido a la historia de vida que construyen continuamente. Dicho abordaje mantiene la expectativa de que los seres humanos desarrollen sus potenciales, cualesquiera que éstos sean. Sin embargo, las dificultades de la actualidad son las que inciden directamente en la "noción que predomina de la persona, al triangularla como eje central de una psicología individualista" (Cupertino, De Rezende y Nisencwajg, 2008) (p. 67). Al asumir al individuo desde otra forma de construcción, se avala una concepción subjetiva, que permite la inclusión de la cultura como eje articulador de su proceso histórico en un escenario social determinado. Entendiendo la subjeti- vidad, como una posibilidad de acuñar diferentes saberes, se expande el tejido social hacia caminos de la diversidad

La diversidad, como una posibilidad de inclusión, supone la creación de nuevos saberes y visiones de mundo. Por lo tanto, la formación académica en psicología así como las intervenciones psicológicas pueden asumirse como oportunidades de transformación, respondiendo a las necesidades propias de los actores. Actores que demandan cada vez más, encuentros menos etiquetados. Es así, como cobra notoriedad el marco de relaciones de fondo, que permitan la construcción de otras formas de vida, sin que atenten con la esencia de cada ser humano.

Los cursos de diversidad cultural por lo general contienen temas que se abordan de manera a veces, somera o aislada a través de los currículos académicos. Los grupos excluidos o rechazados, la discriminación y el prejuicio, la raza, el género, la clase social, la edad, la incapacidad y preferencia sexual y religiosa son algunos de los temas. La formación académica en diversidad cultural debe hacer énfasis en el desarrollo de destrezas y competencias interculturales que posibiliten reconocer los diversos trasfondos, lograr una participación efectiva en la sociedad y asumir una postura ética frente a las diferencias. A su vez debe propiciar una conexión entre lo que se aprende en el salón de clases y la experiencia fuera de éste. Es decir, explorar el contexto universitario y el comunitario.

El estudio de la diversidad cultural en el análisis de la acción humana supone asumir la variedad y las diferencias en las intervenciones e investigaciones psicológicas no únicamente para evaluarlas sino para entenderlas desde el respeto, la tolerancia y la solidaridad. Valores éstos, que propician una mirada crítica, apelando a la consideración de la complejidad humana. Asimismo, la práctica ética y efectiva demanda del psicólogo y de la psicóloga que interviene con poblaciones diversas una postura de autoexploración y crecimiento personal para un entendimiento de la influencia de la cultura en el sistema de creencias y visión de mundo. Una apertura a auto educarse con respecto a la cultura de las personas con quienes interviene. Ir más allá de un acercamiento unidimensional de la cultura y de la gente. Es reconocer la complejidad, multiplici- 
dad y fluidez de las identidades. Supone el respeto a las libertades fundamentales de pensamiento, religión, opinión, expresión y de participar en la vida cultural de su elección.

Asumir una postura de apertura a la diversidad en las prácticas psicológicas es un reto que nos brinda el potencial para el crecimiento personal, la creatividad y una conexión humana profunda. Es una invitación a que debemos aspirar, entre otras cosas, a entender al Otro desde su propia idea de quién es, definido por sí mismo y en su relación con los otros (Molina Carrasquillo, 2006). La tarea, en cierta medida, es pensar a través de los otros. Este pensar a través de los otros supone asumir el reto de un cambio en la manera de pensar, un pensar auto reflexivo, dialógico y por lo mismo de apertura. Ciertamente, desde la psicología hay realidades comunes, sin embargo, la manera de aproximación es diferente, obedeciendo al enfoque que se adopte de dicha realidad

\section{MÉTODO}

Tomando en consideración los méritos así como los supuestos teóricos que se desprenden del propósito de este estudio, el diseño de investigación se basó en un enfoque cualitativo de naturaleza descriptivaexploratoria (Hernández, Fernández y Baptista, 2010). De acuerdo a los autores en los estudios descriptivos el propósito del investigador y de la investigadora es describir fenómenos, contextos, situaciones y eventos. Este diseño permite dar una mirada a los procesos que se suscitan a la vez que se re-conceptúan y re-significan los mismos, desde la concepción de mundo en el que se generan. Esto facilita que a medida que avance el estudio, se vaya delimitando el foco de inquietud. Es decir, se parte de unas interrogantes amplias que se pueden ir refinando y delimitando a medida que progresa el estudio. De igual manera, pueden surgir otros ángulos de interés en el proceso investigativo los cuales son debidamente atendidos. La información que se recopila, provoca una interpretación que recae en los significados que tienen los elementos bajo estudio (Lucca y Berrios, 2009).

El conocimiento científico que se produce no se legitima por la cantidad de elementos bajo estudio, sino por la calidad y descripción de esa expresión. Los ad- jetivos que se le adscriben a esta metodología lejos de pretender establecer generalizaciones y conclusiones absolutas de un fenómeno bajo estudio, propician el escenario para que se profundice en el mismo. Se establece así, una continuidad en la congruencia de los resultados, que hace énfasis en la propia singularidad de lo estudiado. En este sentido, González-Rey (2000) expone que la "epistemología cualitativa se apoya en la significación de la particularidad como nivel legítimo de la producción de conocimiento" (p. 66).

\section{Elementos bajo estudio}

Se examinaron los sílabos de cursos que atienden el tema de la diversidad, en universidades públicas y privadas, que ofrecen estudios de pre-grado en Psicología. De Europa, se contó con la participación de una universidad privada en España. Por parte de Latinoamérica, se sumaron al estudio, dos instituciones de Colombia, ambas privadas.

En el área del Caribe se contó con la representación de Puerto Rico; participaron cuatro universidades privadas y una pública. Una de las universidades atiende el tema de la diversidad en el continuo de tres cursos, mientras que otra, lo concibe en dos. Es importante acotar que de las instituciones que accedieron a participar de la investigación, dos no cuentan con un curso formal de diversidad cultural en su oferta curricular. Sin embargo, el mismo, se aborda a lo largo de varios cursos, por lo que se le realizó una entrevista semiestructurada a la directora y al director de los respectivos programas académicos. La entrevista semiestructurada se caracteriza por ser de corte cualitativo y es útil cuando el asunto de interés de la investigación no está disponible para ser observado. En este caso no se contó con los sílabos de dos instituciones académicas, por lo que se recurrió al recuento oral.

Según Hernández, Fernández y Baptista (2010) la entrevista cualitativa tiene como propósito obtener respuestas para la construcción conjunta de significados respecto a un tema de interés. Los autores puntualizan que la entrevista cualitativa permite recopilar información acerca de un tema, problema o tópico de interés en el lenguaje y desde la perspectiva de la persona entrevistada 


\section{Procedimiento}

Las universidades respondieron al llamado que se hiciera a través de correo electrónico a los representantes de los distintos programas de Psicología. Para salvaguardar la privacidad de la información suministrada por parte de los organismos institucionales, cada uno de los silabarios fue identificado con una letra. Los siete sílabos de Puerto Rico se nombraron con las siguientes letras: A, B, C, D, E, F y G. Asimismo, al documento proveniente de una universidad de España, se le asignó la letra $\mathrm{H}$ y a los sílabos de Colombia, las letras I y J, respectivamente.

Se realizó un análisis de contenido de los sílabos siguiendo el modelo de Wolcott $(2009,1994)$. El autor propone tres componentes: descripción, análisis e interpretación con el propósito de organizar y darle sentido a la información. Mediante estos tres componentes se analizaron los sílabos en cuanto a lo que contenían (descripción), lo que tenían en común y en lo qué se diferenciaron unos elementos con otros (análisis) y por último, el significado y la utilidad (interpretación). De igual manera, las entrevistas-semi estructuradas se analizaron utilizando el modelo de Wolcott

El análisis de contenido se realizó utilizando las siguientes categorías a partir de las preguntas de investigación y la literatura revisada: perspectiva teórica desde la que se aborda el curso, óptica geográfica, contenido del curso, realidades socioculturales e implicaciones psicológicas, congruencia cultural, saberes y maneras de ver, explicar y vivir las realidades que quedan excluidos; fuentes de información y recursos bibliográficos. De la misma manera, se identificó información sensitiva que apelaba a los objetivos de la investigación

\section{RESULTADOS}

\section{Perspectiva Teórica}

El análisis de los sílabos de las universidades bajo estudio, que atienden la diversidad cultural, como parte de la formación psicológica, revela que en uno de ellos, los temas de las bases antropológicas y la dimensión sociológica no están contemplados. Sin embargo, toma como cimiento para la construcción del curso, la relación que cohabita entre la investigación formal y la práctica de la psicología en el país de procedencia. A través del estudio de esta categoría de análisis, se estableció que en su mayoría, los sílabos se han gestado desde las realidades psicosociales y políticas del espacio geográfico en el que se crearon. El sílabo A propone un análisis crítico desde el contexto puertorriqueño, por lo que contempla los cambios y las transformaciones socioculturales que han devenido a partir del siglo XX. El sílabo D toma en consideración los antecedentes históricos, así como, las manifestaciones de la cultura puertorriqueña, para entender el proceso de aculturación de la sociedad contemporánea.

En el sílabo H, se hace énfasis en concebir la diversidad cultural como un curso de la formación psicológica, atravesado por una constante revisión de los procesos que se generan en la sociedad. Se plantea en el mismo que si la asignatura analiza la "diversidad" del comportamiento humano deberá, entonces, brindar apoyo teórico para las intervenciones ante la "adversidad". La óptica de este curso nos remite a la postura de García-Roca (2001), en la que la Academia está llamada a jugar un papel decisivo en la erradicación actual de la exclusión, tanto para reducir su alcance como para ampliar la ciudadanía social. Ciertamente, se genera el espacio para el enriquecimiento de la configuración humana, lo que repercute en un esfuerzo por resolver los conflictos que plantean las transformaciones sociales aceleradas. Pues, es a partir de la comprensión de la diversidad humana, que no se descuida la unidad múltiple de los universales antropológicos, situados principalmente, en la estructura compleja y organizadora de la interrelación biológica, psicológica y sociocultural;

\section{Óptica Geográfica}

En el sílabo de la universidad I, se postula que el modo de concebir la diversidad debe apelar a los conflictos socioculturales que han ganado espacio en dicho contexto geográfico. Cabe mencionar que, aunque no se expone de manera explícita en el sílabo, la situación local de ese país se ha descrito en torno a los problemas de exclusión social, generando un deterioro en la convivencia ciudadana. Hoy, se podría agregar al panorama, "la agudización del conflicto armado, el desplazamiento, las desapariciones y los secuestros" (Robledo, 2008) (p. 10). La manera de hacer Psicología en ese espacio territorial, responde a las diferentes formas de vida que se tejen entre sí. 
Este planteamiento propicia que desde la Academia se posibilite el enriquecimiento de perspectivas variadas y se potencialice la transformación del mundo contemporáneo, atravesado por el intercambio social que se genere. Ciertamente, el compromiso que se tiene con la profesión, contribuye a la construcción de un proyecto de mundo, que respete los derechos inalienables de igualdad y libertad.

Los conceptos de justicia e igualdad dentro del contexto de la educación formal en el continente europeo se asumen desde el análisis y la evaluación de las distintas idiosincrasias de los colectivos y personas que conviven en ese espacio territorial. Torres (2008) expone que "Europa es una comunidad en sí misma pluricultural, por lo que se trata de asumir la diversidad como un valor enriquecedor de la humanidad y concebir una educación abierta a las diferentes culturas" (p. 87). El sílabo H establece que la diversidad, como asignatura, responde al cambio de la cultura docente que han desarrollado en los últimos años. Es por eso, que las transformaciones surgidas se enmarcan bajo el Nuevo Espacio Europeo de Educación Superior, lo que supone un modo diferente de entender la educación universitaria. Puesto que, se hace imperativo que tal formación sea útil al profesional de la Psicología en el andamiaje de su ser, como persona. En esa dirección el estudiantado:

Por una parte, va a estudiar las diferencias grupales inicialmente derivadas a variables biológicas como el sexo. Por otra, va a analizar las diferencias organizadas en torno a las variables socioculturales, como es el caso de la exclusión social. Por último, el alumno tendrá la posibilidad de analizar los procesos de comunicación intercultural y profundizar en la realidad de la inmigración en nuestro país.

$\mathrm{Al}$ adentrarse al contexto caribeño, sin duda alguna, es fundamental entender que el mismo, no se trata de un todo homogéneo. Menjívar (2006), acota que esto es comprensible por la "diversidad de sujetos sociales involucrados y su continua respuesta a situaciones cambiantes" (p. 94). En el proceso del análisis de los sílabos provenientes de las diferentes universidades de Puerto Rico, se aprecia un marcado interés por acuñar las transformaciones en el contexto sociocultural local. Esto incide de manera directa en el estudio del impacto de la cultura puertorriqueña. El sílabo A, considera las intervenciones e investigaciones psicológicas para analizar el impacto de la globalización, la tecnología y la cibernética en el país. Sin embargo, sólo uno de los sílabos estudiados no contempla el impacto histórico en el modo de hacer psicología.

Ciertamente, hay un consenso respecto a cómo se concibe la óptica geográfica al momento de diseñar cursos que apelan a la diversidad cultural. Pues, la misma se representa en los sílabos principalmente como una visión local. Visión que se arraiga en los postulados de Ignacio Martín Baró (1987), quien invitaba a examinar los problemas específicos que se generan en los pueblos para adueñarse del conocimiento psicológico de las realidades de América Latina. Cabe mencionar que no todos los sílabos exponen de manera explícita un enfoque global. En varios se aborda como tema, pero no como óptica para enmarcar el curso.

\section{Contenido del Curso}

Las distintas universidades de las que proceden las propuestas curriculares que forman parte de este estudio, toman en cuenta los postulados en los que sostienen sus proyectos educativos. Algunas, reconocen que se han debatido entre las propuestas que avala la American Psychological Association en cuanto a formar investigadores basándose en la evidencia científica, y los lineamientos señalados por la Federación Europea de Asociaciones de Profesionales de Psicología (Robledo, 2008). Esto permite el acercamiento hacia la pluralidad de enfoques, manteniendo disposición a la apertura del trabajo interdisciplinario. Dicho planteamiento descansa en una integración de los supuestos de ambos organismos, contrario a Europa, Estados Unidos y Puerto Rico (por su situación política), que deben regirse por agencias acreditadoras que dictan la norma de la educación a seguir en los programas de Psicología. Esto, para contar con el prestigio y reconocimiento a nivel internacional.

"Varias iniciativas de la American Psychological Association, proveen evidencia de la importancia de la diversidad, esto incluye cambios recientes al Código de Ética y la creación del Comité de Acreditación a programas graduados de Psicología" (Green, Callands, Radcliffe, Luebbe y Klonoff, 2009) (p.1057). El estu- 
dio de percepción llevado a cabo por Green et al., (2009), utilizando estudiantes de psicología clínica, expuso que la concepción que se tiene de la diversidad, "se focaliza primordialmente a la etnicidad, raza, cultura y género" (p.1066). Es decir, desligan de su entrenamiento, conceptos de fondo como: discapacidad, nacionalidad, orientación sexual, educación o el lenguaje. Dentro de los países participantes de esta investigación, hay que destacar que Puerto Rico, merece especial atención en este asunto, ya que por su estatus colonial, requiere una sensibilidad política y cultural distinta. Los contenidos curriculares de dicho país, escasamente incorporan temas de preocupación política. De los siete sílabos estudiados, sólo dos, abordan este aspecto relevante.

Ciertamente, el abanico temario es uno extenso y variado, sin embargo, ocho sílabos apelan al estudio de la práctica psicológica. Las categorías de raza, etnia y clase social se contemplan en cuatro sílabos. Igualmente, en cuatro escritos toman en consideración el género y la orientación sexual. De otra parte, sólo dos sílabos abordan la identidad religiosa de las personas. Es pertinente acotar que hay una tendencia en los diferentes países a asumir la identidad nacional, la realidad política y cultural de esos contextos como contenidos imprescindibles de los cursos. Es pertinente señalar que los cursos examinados hacen énfasis en la diversidad cultural (desde cultura como social). Esto, podría limitar el estudio o acercamiento de temas como asuntos de género, orientación sexual y prácticas religiosas entre otros, que bien podrían formar parte del contenido de un curso de diversidad humana.

\section{Realidades Socioculturales e Implicaciones Psi- cológicas}

La inclusión de las realidades socioculturales y las implicaciones en el quehacer psicológico, se presentan de manera numerosa en los silabarios examinados. Las realidades de mayor interés resultaron ser: repercusión del sistema de valores en la práctica psicológica, destacado en cuatro sílabos, y el racismo, etnicidad y religión, abordados por cinco escritos. No obstante, la diversidad cultural se ha acrecentado en muchos lugares del mundo a partir de la compleja situación de las corrientes migratorias. Este hecho es tomado en consideración en tres de los sílabos analizados. Uno de éstos puntualiza: "la labor Universitaria en su conjunto no puede ni debe quedarse al margen de los marginados, sino que está llamada a jugar un papel decisivo en la erradicación actual de la exclusión, tanto para reducir su alcance como para ampliar la ciudadanía social" (García Roca, 2001: p. 5), por la sencilla razón de que es un hecho real, irracional e injusto.

\section{Congruencia Cultural}

La diversidad trae consigo arduos retos y muchos de éstos son pertinentes al campo de la Psicología. Es por eso, que se hace énfasis en el análisis minucioso de los currículos de la disciplina a nivel superior. Pues, deben responder a las necesidades de una sociedad culturalmente diversa. Thomas (2004) sostiene que "el adiestramiento de los psicólogos y psicólogas debe contemplar la sensibilidad cultural y el entendimiento de la diversidad" (p. 105). El interés de mirar esta categoría de análisis dentro del estudio, responde a auscultar hasta qué punto, el contenido de los sílabos apela a las realidades socioculturales de los respectivos países. Puesto que entender la dimensión psicológica del ser humano supone atender la mirada del tejido social que intenta dar cuenta de su complejidad.

Las condiciones de vida en Puerto Rico, aluden a un contexto colonial, este asunto es abordado en tres universidades, no así, en dos instituciones del país. Es posible que esto responda a que el tema se obvia por entender que no es materia concerniente al quehacer psicológico. Ciertamente, es un asunto que apela a lo político, por lo que asumirlo, propicia el surgimiento de cuestionamientos ideológicos.

En los sílabos examinados de las instituciones de España y Colombia, el tema no se contempla, lo que es comprensible, dado que no responde a su contexto social. Sin embargo, el país europeo aborda las implicaciones migratorias, acercándose a los significados y construcciones que se interpretan de las experiencias. De otra parte, el sílabo de una de las universidades colombianas, asume el conflicto armado como una realidad nacional. Se aprecia además, preocupación por las presiones sociales hacia la adaptación y la aculturación, evidenciado en seis de los siete sílabos examinados, y la intervención psicológica en cuatro de las universidades. 
Esta categoría, también permite ver que una universidad puertorriqueña focaliza en los cambios en percepciones y valores atravesados por la historia. El sílabo $\mathrm{H}$, profundiza en los fenómenos que explican las bases sociales de la conducta y contempla el debate entre la cultura (nivel micro) y el psiquismo humano (nivel humano). Por su parte, el sílabo I, extrapola la relación cultural con las investigaciones referentes al componente familiar y los conflictos que del mismo se generan. Ciertamente, esta categoría de análisis establece que los asuntos sociopolíticos no pueden desligarse de las implicaciones del entramado psicológico. Parafraseando a Martin Baró (1983), no somos seres arrojados al vacío pues, estamos ligados a la historia que se teje en redes de múltiples vinculaciones sociales.

\section{Saberes y maneras de ver, explicar y vivir las realidades que quedan excluidos}

En la revisión de los sílabos, se identificaron varios temas que no se abordan de manera prominente. Entre estos, destacan asuntos relacionados a la dimensión histórica: colonialismo, globalización y tecnología, así como, temas concernientes a la diversidad humana: género, orientación sexual y diversidad religiosa. Una posible explicación a estas posturas, puede deberse a que quizá, responden a los principios dictados por las universidades de procedencia o a las exigencias de las agencias acreditadoras. Esto se alinea a posturas paralelas que contemplan una visión del panorama local, dejando de lado una referencia de apertura al plano mundial y virtual. Por otra parte, en uno de los sílabos analizados, proveniente de Latinoamérica, se evidencia la consideración de otros procesos accionales que apelan a la construcción de realidades contribuyendo así a la emergencia de distintas identidades. Identidades que ciertamente responden a nuevos estilos de vida, desde distintos ideales, tradiciones y maneras en que se explican los fenómenos.

"En el reconocimiento de la importancia de una formación holística que responda a las necesidades del nuevo siglo y a sus procesos socioeconómicos, emerge la exaltación de la cultura en los procesos de conformación social y construcción de la identidad. ...concientizar a los estudiantes acerca de la existencia de comportamientos etnocéntricos y percepciones limitadas en la interpretación de las diferencias culturales, y que dificultan el trabajo conjunto con otras culturas y grupos sociales a causa de la imposibilidad de entender y dialogar con otras formas de ver el mundo, ideales, costumbres y formas de vida."

\section{Fuentes de Información}

Los sílabos revisados comparten el interés por incorporar fuentes de información que abordan la diversidad en el contexto de la intervención psicológica. Congruente con el curso, se enriquecen de los desarrollos teóricos y metodológicos de disciplinas como la historia, la antropología, sociología, la ética, la literatura y la etnografía. Como se señala en el sílabo $\mathrm{H}$ por ejemplo, el curso de diversidad presenta "un amplio abanico de posibilidades y contenidos que se entremezclan con diversas disciplinas y enfoques".

Incluyen además, artículos de corte investigativo, presentaciones, ponencias en congresos y actividades profesionales. Asimismo, aparecen como referencias, artículos cuyos temas no están explícitos en el sílabo. Cabe mencionar que el enfoque presentado en uno de los contenidos curriculares, se circunscribe a una plataforma virtual, por lo que la convergencia de conocimiento, se da a través de foros de discusión. Todo lo anterior, se centra en el complejo análisis entre las realidades socioculturales y sus implicaciones psicológicas.

\section{DISCUSIÓN}

El reconocimiento a nivel mundial de la diversidad cultural, puede destacarse como un fenómeno de carácter social, evocado desde finales del siglo XX. Ciertamente, se trata de un constructo que no es nuevo, por el contrario, ha estado presente a lo largo de la historia de la humanidad. Roa (1995) articula que el discurso de la postmodernidad ha venido a reconocer y valorar la diversidad como un rasgo constitutivo de las sociedades actuales. Esto supone un importante cambio de perspectiva en los imaginarios a nivel colectivo, de aquellas sociedades que por siglos han aunado esfuerzos para tratar de ignorar todo aquello que la cultura dominante no puede explicar. Sin embargo, es propio destacar que frente a posturas impositivas que intentan exterminar aquellas expresiones que no se ajustan a los cánones del 
dictamen oficial, surgen movimientos nacionalistas y corrientes intelectuales que destacan la importancia de preservar todas las formas de expresión de los pueblos. Pueblos que advienen desde los grupos humanos, a partir de los cuales se construye la riqueza cultural diversa y sus legados generacionales.

En un mundo que tiende a resaltar la inequidad, la desigualdad, la fragmentación y las situaciones de exclusión y discriminación se yuxtaponen retos que se encaminan hacia la asimilación de un discurso hegemónico, conjugado con la pobreza, conflictos, guerras y desastres que impactan directamente a la persona. El entrecruzamiento complejo de diversas poblaciones, repercute a su vez, en el advenimiento de problemas psicosociales y políticos. Es así, como las sociedades ejercen un rol excluyente hacia las personas por asuntos que se salen de la norma dictada, ya sea de índole religioso, económico, de género, entre otros aspectos. Esto, incide directamente en el atropello de espacios que apelan a la transformación social, lo que a su vez, da paso a la perpetuación de un sistema educativo que redunda en lo tradicional y esboza un contenido que subyace a partir de la homogeneidad. De esta manera, se perpetúa un estilo de segregación y exclusión.

Ante tal exposición, se ha generado una preocupación por impulsar planteamientos de fondo, que aboguen por caminos de integración social y que garanticen la participación activa de los actores en la vida pública. Estas corrientes se nutren en el reconocimiento de los seres humanos, como sujetos de derecho para la creación de movimientos inclusivos. (Naciones Unidas, 2001; Torres, 2008). Con ese fin, se sugiere la promoción de capacidades y conceptos en aquellas personas que reciben una educación formal, tales como el conocimiento histórico, el espacio geográfico en el que están situados y la actitud de moverse en contextos diferentes. Robledo (2008) acota que "para hablar de los procesos de formación en una disciplina en particular, en este caso la Psicología, resulta necesario hacer una breve contextualización del país en el cual se vive" (p. 10).

En la actualidad, desde la mirada de las sociedades contemporáneas, el reto ante la diversidad se percibe con gran complejidad. Se destaca que este constructo se imbrica en un área de especial atención, la educación. Y es a través del propio sistema educativo, que se ha venido afianzando un movimiento que intenta dar respuesta a la multiculturalidad que cohabita en los diferentes espacios sociales. Como afirma García (2007), la educación institucionalizada fue pensada para "la selección y homogenización cultural, lo que supone derribar fuertes y arraigados pilares de historia" (p. 96). Este supuesto apoya fielmente los lineamientos que consideran la diversidad como una manifestación natural de la individualidad humana y no como un déficit a corregir.

Si bien la diversidad se ha adentrado fuertemente a los cánones de la historia, su operacionalización ha sido motivo del fragor de intensos debates, como se ha reseñado a lo largo de este escrito. Cabrerizo y Rubio (2007) la conciben como una característica propia de la condición humana, que se manifiesta en el comportamiento y modo de vida de los individuos, así como, en sus modos de pensar, por lo que su presencia llega a todos los niveles evolutivos y a todas las situaciones. Prado (2004) por su parte, se sitúa en el paradigma de la postmodernidad y hace referencia a individualizar, compensar y promover igualdad de oportunidades, consideradas como relevantes, desde el punto de vista de la circunstancia de ser diferente. Turra (2009) resalta como una "verdad incuestionable que todas las sociedades son multiculturales en función de sus complejos desarrollos históricos" (p. 38).

El ámbito universitario, por su parte, se ha convertido en un espacio donde se encuentran miembros de diversos orígenes culturales. En la actualidad, el alumnado desarrolla sus estudios ante una nueva realidad social más multicultural. Sin lugar a dudas, las relaciones interculturales de estas personas en el contexto académico, traspasan las fronteras y se insertan marcadamente al ámbito cotidiano. De ahí, García y Saura (2008) exponen que "la percepción del otro de manera positiva, junto con el rechazo a toda actitud y comportamiento intercultural negativa, deba ser favorecida bajo los principios de la igualdad y el respeto a la diferencia" (p. 72).

Ahora bien ¿Qué acciones nos moverían a trascender las fronteras geográficas y teóricas y hacer visibles otros saberes y maneras de ver, explicar y vivir 
las realidades? Se hace imprescindible emprender acciones y escuchar las voces que faciliten el proceso de inclusión entre las personas inmigrantes y nacionales. La superación de la mirada culturalista como asignatura pendiente, que atribuye la mayor parte de las diferencias entre las personas a sus lugares de procedencia, debe emprenderse. El lugar de origen es una característica, como cualquier otra, de los seres humanos, que influye, pero no determina sus necesidades, deseos, capacidades o cualidades personales. (Prado, 2004). La diversidad y la diferencia son intrínsecas a las personas y sus sociedades; ya existían y seguirán existiendo. La inclusión se hace posible desde la promoción de una identidad cívica y colectiva que albergue tanto los intereses y tareas comunes como las diferencias. (García, 2007)

Esta era histórica, nos propone una mirada a la complejidad social, que nos obliga ampliar nuestra óptica teórica y geográfica para entender las realidades que vivimos como sujetos. Los estudios universitarios y las prácticas como académicos y profesionales por lo general, se sostienen en un cuerpo hegemónico que responde a una cultura en particular con pretensión universalista (Miranda, 2006). La Psicología se enfrenta a una ardua tarea, al reconocer que no existe una perspectiva única, al abordar la diversidad como una realidad. Sin embargo, se debe admitir que las pretensiones universitarias por homogeneizar los saberes, coexisten con el momento histórico en que se intenta dar cuenta del fenómeno que acontece. En este proceso pueden quedar invisibilizados saberes y maneras que pretenden ver, explicar y vivir las realidades. La existencia de la disciplina, en este universo educativo paradójico nos reclama el reconocimiento de ese escenario complejo, como el primer paso para desarrollar estrategias que cambien ese panorama.

La diversidad cultural pasa por un momento histórico en que se constituye una posibilidad epistemológica legítima. Es así, que se concibe como un patrimonio a ser respetado y preservado. Diversas corrientes educativas comienzan a otorgar espacios en sus currículos para atender este fenómeno sociocultural (Humphrey \& Geary, 2005; Torres, 2008). La Academia asume una postura de importancia, al propiciar una educación sensible a las diferencias que confluyen. Se espera que los psicólogos y las psicólogas se muevan en un adiestramiento que reconozca la pertinencia de las diferencias individuales que se generan en los contextos sociales. Desde esa óptica del entendimiento, la Psicología no puede hacerle un flaco favor a la profesión ni debe estar a merced de una sociedad que legitime el bienestar de los menos sobre los más. Ciertamente, asumir las diferencias implica reconocer la importancia de posturas diversas, no desde el obstáculo o el déficit sino, desde el enriquecimiento de los distintos saberes. Es inevitable rechazar la existencia de prácticas hegemónicas, pero a la vez, es necio negar los movimientos que dan mayor apertura a la inclusión. En el fragor de la construcción social de las realidades, es ineludible atender las dimensiones de la diversidad humana dentro de una perspectiva más global e inclusiva y menos local o regionalista. De no hacerlo corremos el peligro de caer en las mismas posturas que pretendemos evitar. Por lo que debe incorporar en los cursos de diversidad los postulados filosóficos que den cuenta de la complejidad y profundidad de la naturaleza humana así como, de los factores que atraviesan culturas y creencias para unificar y no dividir (desde la óptica ética, moral, teocéntrica y utilitaria).

Entendemos la importancia del cuestionamiento y la auto-reflexión de los alumnos como personas ancladas en contextos socioculturales e históricos multidimensionales. Es por eso, que los cursos deben propiciar que los estudiantes se entiendan a sí mismos y a los compañeros y compañeras de clases por medio de la discusión y análisis de situaciones o eventos que les evoquen responsabilidad social. A su vez, los docentes debemos dar una mirada a las propias actitudes y creencias para lograr cambios en los futuros profesionales. Propiciar este análisis personal incluye el reconocimiento de nuestros procesos de internalización y socialización y la necesidad de un marco de respeto hacia los demás. Urge trasladar ese autoconocimiento al plano de las competencias profesionales, pues no nos parece suficiente el conocimiento de la diversidad si está vacío de convicción y actitud de respeto a la igualdad y dignidad de la persona humana.

Elicker, Snell y O’Malley (2010), argumentan que "hay una necesidad imperiosa por parte del académico, 
de infundir contenidos multiculturales en sus cursos para incrementar su pertinencia en la vida de los estudiantes de psicología" (p. 39). De igual manera, Cupertino, De Rezende y Nisencwajg (2008) sostienen que al "promover el debate de prácticas innovadoras y creativas, dirigidas tanto al cuidado de la población como a la formación de los psicólogos y las psicólogas propicia la confrontación de distintos saberes" (p. 65). Por lo tanto, en los diferentes abordajes en que se enmarca la psicología, también cohabita la diversidad. Ciertamente, las personas que solicitan atención psicológica son diferentes entre sí, por lo que cualquier intento de homogeneizar el entendimiento humano, limitaría el enriquecimiento intrínseco de la variedad.

El curso de diversidad humana debe convertirse en un seminario de exploración y crecimiento personal, de entendimiento de la influencia de la cultura en el sistema de creencias y visión de mundo, de exposición y apertura a las diferencias que puedan existir entre las personas. Los sílabos deben ser revisados y atemperados a los cambios histórico-sociales que surjan. Se hace necesario la revisión de lo que se enseña, cómo se enseña y de provocar responsabilidad social y valores democráticos (Giroux, 1994). Esto supone que la disciplina psicológica se posicione en diálogos concertados que permitan la consideración de la realidad contextual de sus actores. Es imperativo trabajar desde procesos cognoscitivos hasta emotivos, para lograr mayor nivel de sensibilización personal. Asumir actitudes saludables al quehacer profesional indistintamente de la población a la cual nos toque servir, es un deber.

\section{REFERENCIAS}

Cabrerizo, J. \& Rubio, M.J. (2007). Atención a la diversidad. Teoría y práctica. Madrid: Pearson.

Cupertino, C., De Rezende, C. \& Nisencwajg, G. (2008). Hacia un quehacer psicológico creativo: la preparación para la atención a la diversidad. Revista de Psicología, 26 (1), 64-91.

Elicker, J., Snell, A. \& O’Malley, A. (2010). Do student perceptions of diversity emphasis relate to perceived learning of psychology? Teaching of Psychology, 37 (1), 36-40. doi: 10.1080/ 00986280903425706

García, A. \& Saura, J. (2008). Estudio de las consecuencias de la diversidad cultural en espacios de encuentro pluricultural. Revista Española de Lingüística Aplicada, 21, 71-84.

García, J. (2007). Multiculturalidad y educación: una discusión pedagógica y epistemológica para el siglo XXI. Revista de Filosofía de la Universidad de Costa Rica, 45, 93-98.

García-Roca, X. (2001). Universidad y acción solidaria. Documento no publicado.

Giroux, H. (1994). Doing Cultural Studies: Youth and the Challenge of Pedagogy. Harvard Educational. 64, (3), 278-309.

González-Rey, F. (2000). Lo cualitativo y lo cuantitativo en la investigación de la psicología social. Revista Cubana de Psicología, 17, (1), 6171.

Green, D., Callands, T., Radcliffe, A., Luebbe, A. \& Klonoff, E. (2009). Clinical psychology students' perceptions of diversity training: a study of exposure and satisfaction. Journal of Clinical Psychology, 65 (10), 1056-1070. doi: 10.1002/ jclp.20605

Hernández, S., Fernández, R. y Baptista, C. (2010). Metodología de la investigación. (5ta Ed.). Mexico: Mc Graw Hill.

Humphrey, D. \& Geary, C. (2005). Curricular change gains momentum: New requirements focus on diversity and social responsibility. Recuperado el 9 de noviembre de 2005 de Diversity Digest file:/ /C:/Documents and Settings Administrator/ Desktop/new requirement focus on diversity.

Lucca, N. \& Berríos, R. (2009). Investigación cualitativa: fundamentos, diseños y estrategias. Ediciones SM.

Martín-Baró, I. (1987). Retos y perspectivas de la psicología en América Latina. Conferencia pronunciada en la Universidad Javeriana en el XXV aniversario del establecimiento de su facultad. Bogotá. 
Martín-Baró, I. (1983). Acción e ideología. Psicología social desde Centroamérica. El Salvador: UCA.

Martínez, M. F. (2006). Diversidad cultural: una oportunidad y un desafío para la convivencia. Revista Electrónica de Psicología Científica, 1-10.

Menjívar, M. (2006). Culturas en movimiento. Diáspora, esclavitud y diversidad cultural en el Caribe colonial. Revista Reflexiones, 85, (1-2), 93106. 15

Miranda, D. (2006). Hacia una conceptuación de la diversidad. Departamento de Psicología. Universidad de Puerto Rico. Documento no publicado.

Molina Carrasquillo, R. (2006). ¿Por qué hablar de diversidad? Departamento de Psicología. Universidad de Puerto Rico. Documento no publicado.

Naciones Unidas (2001). Declaración Universal de la UNESCO sobre la Diversidad Cultural. Recuperado el 5 de septiembre de 2010 de http:// www.unesco.org/culture/pluralism/diversity/html sp.index/sp.shtml

Ortiz, H. (2007). Formación del profesorado y atención a la diversidad: desafíos a los procesos de innovación curricular en educación inicial. Revista Académica de la Universidad Católica del Maule, $33,45-60$.
Prado, J. (2004). La igualdad educativa en la interculturalidad: hacia un perfil específico de la diversidad en educación física. Revista de Educación, 10 (72).

Roa, A. (1995). Modernidad y postmodernidad. Santiago de Chile: Andrés Bello.

Robledo, A.M. (2008). La formación de psicólogas y psicólogos en Colombia. Universitas Psychologica, 7, (1), 9-18.

Thomas, T. (2004). Psychology in a culturally diverse society. Australian Psychologist, 39, (2), 103-106.

Torres, J. (2008). Diversidad cultural y contenidos escolares. Revista de Educación, 345, 83-110.

Turra, O. (2009). Diversidad cultural en el currículo: Un discurso educacional que posibilita la consideración de las formas culturales mapuche en los contenidos escolares. Horizontes Educacionales, 14, (1), 37-49.

Wolcott, H.F. (2009). Writing Up Qualitative Research. (3th Edition). California: Sage Publications, Inc.

Wolcott, H.F. (1994). Transforming qualitative data: Descriptions, analysis and interpretation. California: Sage Publications, Inc. 